A Modified Direct Method for the Calculation of Elastic Moduli of Composite Materials

\author{
J. A. Wang \\ Oak Ridge National Laboratory* \\ P.O. Box 2008 \\ Oak Ridge, TN 37831-6370 \\ J. Lubliner and P.J.M. Monteiro \\ University of California \\ Berkeley, CA 94720
}

RECENED

FEB 05 1985

OSTI

Presentation to the First International Conference on Composites in Infrastructure (ICCI'96), Tucson, AZ, January 15-17, 1996; to be published in the Proceedings.

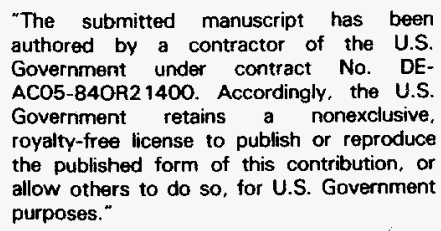

*Managed by Lockheed Martin Energy Systems, Inc., under contract DE-AC05-84OR21400 with the U.S. Department of Energy. 


\title{
A MODIFIED DIRECT METHOD FOR THE CALCULATION OF ELASTIC MODULI OF COMPOSITE MATERIALS
}

\author{
J. A. Wang \\ Computational Physics and Engineering Division \\ Oak Ridge National Laboratory \\ Oak Ridge, TN 37831-6370 \\ J. Lubliner and P. J. M. Monteiro \\ Department of Civil Engineering \\ University of California \\ Berkeley, CA 94720
}

\begin{abstract}
The modified direct method is a scheme for the estimation of elastic moduli of composite materials and is based on micromechanical theory and classical elasticity. Using the statistical homogeneous assumption and the two-phase composite approach, one takes the average field of the composite. Due to the complexity of composite materials, the modeling parameters for the exact analytical theory are not always available and then the effective bounds are usually too wide for practical application. For engineering purposes a more practical and general model is desired. The modified direct method was developed to approach the above requirements. In this work the modified direct method is compared with different available experiment data and methods, for example, Kuster-Toksöz, Christensen-Lo. The comparison results show that the modified direct method provides a very good estimation of the elastic moduli in different kinds of problems, such as the soft and hard inclusion cases, porous materials, at various concentrations and/or various porosities.
\end{abstract}

Keywords: modified direct method, elastic moduli, composite materials, two-phase composite, aggregate composite, nondestructive test, ultrasonic test, porous materials.

\section{Introduction}

A composite material consists of a number of distinct homogeneous phases that form regions large enough to be regarded as continua and that usually bond together at the interface. Many natural and artificial materials are of this nature, such as some rocks (for example, sandstone), filled polymers, mortar, concrete, precipitate, porous and cracked media, etc. The development of mathematical models for composite materials is an extremely complicated and difficult task because of the large number of factors on which the behavior of the composite depends, such as phase factors [1-3], composition factors [4-6], and operational factors [7]. In order to develop a rigorous model for a composite, these factors must be under control, either by means of a particular model describing their influence or by suitable assumptions. 
From a practical point of view, two kinds of information determine the properties of a composite material: the internal phase geometry [8] (i.e., the phase interface geometry) and the physical properties of the phase (i.e., the constitutive relation of these); the former is far more difficult to classify than the latter. Since the early 1960s, there has been much work on the elastic properties of heterogeneous materials [9-12]. Much of this work is of a fundamental nature and provides a sound basis for understanding and discussing the properties of aggregates and composites. The purpose of this research is to present a direct method that was developed for the estimation of elastic moduli of composite media. In Section 3, the modified direct method is compared with other schemes, and its predictions are verified using existing available experimental data. In Section 4, experimental research is conducted on the effect of porosity on the elastic moduli of cement paste and on the amount of sand on the elastic moduli of mortar. It is shown that the proposed model predicts values that are in good agreement with experimental results.

\section{General Considerations}

Two important assumptions are usually made when treating the macroscopic elastic properties of composite materials: statistical homogeneity and statistical isotropy [13]. For the problem to be workable, a necessary characteristic of a composite material is statistical homogeneity (S.H.). This assumption essentially means that large enough subregions of the composite are statistically identical with the whole sample. The most frequently considered subregion of the multiphase medium is the two-phase composite in which one phase may be regarded as matrix in which arbitrarily shaped inclusions of other phases are embedded.

The effective properties of a composite material, when their space variation is statistically homogeneous, define the relations between averages of field variables, such as stress and strain. To produce a statistically homogeneous field in a composite, it is expedient to apply boundary conditions that produce homogeneous fields in a homogeneous body. Such boundary conditions will consequently be called iso-strain or iso-stress. For elastic bodies, iso-strain or iso-stress conditions are either one of the following:

$$
u_{i}(S)=\varepsilon_{i j}^{0} x_{j} \text { or } T_{i}(S)=\sigma_{i j}^{0} n_{j}
$$

where $\varepsilon_{i j}^{0}$ are constant strains and $\sigma_{i j}^{0}$ are constant stresses. The effective elastic properties are defined by the linear relations:

$$
\bar{\sigma}_{i j}=\bar{C}_{i j k l} \bar{\varepsilon}_{k l}, \bar{\varepsilon}_{i j}=\bar{C}_{i j k l}^{-1} \bar{\sigma}_{k l}
$$

where $\bar{C}_{i j k l}$ is the effective elastic moduli tensor and $\overline{\boldsymbol{\sigma}}_{i j}, \bar{\varepsilon}_{i j}$ are the average stresses and strains, respectively. When $u_{i}(\mathcal{S})=\varepsilon_{i j}^{0} x_{j}$ is prescribed, then $\bar{\varepsilon}_{i j}=\varepsilon_{i j}^{0}$.

The evaluation of an average elastic modulus of a composite material or polycrystals 
is one of the classical problems in micromechanics. The pioneering works have been done by Voigt and Reuss. The Voigt approximation gives upper bounds, and the Reuss approximation gives the lower bounds of the average elastic modulus as prove by Paul [14] and Hill [15]. The computation of effective properties in terms of the average will be called the direct approach. In general, it requires determination of the appropriate fields in the phases as defined by the field equations, interface continuity conditions, and external homogeneous boundary conditions in order to compute the required average.

\section{Development of the Modified Direct Method}

\subsection{Introduction}

Let us consider a composite material where the domain $\Omega$ includes inclusion, $\Omega_{r}$, and matrix, $\Omega-\Omega_{r} . \mu, K$ are, respectively, the shear and bulk modulus of the matrix, and $\mu_{r}, K_{r}$ are, respectively, the shear and bulk modulus of the inclusion. The prescribed iso-strain condition $\varepsilon_{i j}^{0}$ is impressed throughout the medium, and $\sigma_{i j}^{0}$ is the corresponding stress field. The displacement field is then $u_{i}^{0}=\varepsilon_{i j}^{0} x_{j}$. Due to the inhomogeneity, there will be disturbance displacement field, $u_{i}$, and stress fields, $\sigma_{i j}$, in the domain $\Omega$. The total displacement and stress fields are $u_{i}^{0}+u_{i}$ and $\sigma_{i j}^{0}+\sigma_{i j}$, respectively.

In this analysis, we use the spherical shape inclusion (from the Eshelby result [16], the stress field in the spherical shaped inclusion is uniform), and we assume that the phases are ideally elastic and homogeneous over the whole of their bulk. Also the adhesion between phases is perfect and any other form of interaction is absent. The stress components are self-equilibrated, that is, $\sigma_{i j, j}=0$ and $\sigma_{i j}=0$ at infinity. When a finite body is considered, then $u_{i}(S)=0$ at the boundary $S$ for the prescribed iso-strain boundary condition. According to the equivalent homogeneous inclusion method [16], there will be an eigenstrain in the inclusion which has the same $\mu$ and $K$ as the matrix. This causes the same effect as the inhomogeneity in the matrix. Now, Hooke's Law can be written as:

in $\boldsymbol{Q}$,

or

in $\mathbf{Q}-\mathbf{\Omega}$

$$
\sigma_{i j}^{0}+\sigma_{i j}=C_{i j k l}^{*}\left(\varepsilon_{k l}^{0}+\varepsilon_{k l}\right)
$$

$$
\sigma_{i j}^{0}+\sigma_{i j}=C_{i j k l}\left(\varepsilon_{k l}^{0}+\varepsilon_{k l}-\varepsilon_{k l}^{*}\right)
$$$$
\sigma_{i j}^{0}+\sigma_{i j}=C_{i j k l}\left(\varepsilon_{k l}^{0}+\varepsilon_{k l}\right)
$$

$$
\sigma_{i j}^{0}=C_{i j k l} \varepsilon_{k l}^{0}=C_{i j k l} \mu_{k, l}^{0}
$$

$\varepsilon_{k l}^{*}$ is the eigenstrain tensor (referred to by Eshelby as stress-free transformation strain) 
in $Q_{r}$, and $C_{i j k l}$ is the elastic stiffness tensor of the inclusion.

\subsection{The Direct-Solution Scheme}

The necessary and sufficient condition for the equivalency of strain and stress in Eqs. (3.1) and (3.2) is:

in $\mathbf{Q}$,

$$
C_{i j k l}^{*}\left(\varepsilon_{k l}^{0}+\varepsilon_{k l}\right)=C_{i j k l}\left(\varepsilon_{k l}^{0}+\varepsilon_{k l}-\varepsilon_{k l}^{*}\right)
$$

or, for an isotropic material,

$$
2 \mu^{*}\left(\varepsilon_{k l}^{0}+\varepsilon_{k l}\right)+\lambda^{*} \delta_{i j}\left(\varepsilon_{k k}^{0}+\varepsilon_{k k}\right)=2 \mu\left(\varepsilon_{k l}^{0}+\varepsilon_{k l}-\varepsilon_{k l}^{*}\right)+\lambda \delta_{i j}\left(\varepsilon_{k k}^{0}+\varepsilon_{k k}-\varepsilon_{k k}^{*}\right)
$$

Here $\varepsilon_{k l}$ can be obtained as a known function of $\varepsilon_{k l}^{*}$ when the eigenstrain problem in the homogeneous material is solved. Thus Eq. (3.6) determines $\varepsilon_{k l}^{*}$ for a given $\varepsilon_{k l}^{0}$ in such a manner that equivalency holds so that after obtaining $\varepsilon_{k l}^{*}$ the stress $\sigma_{i j}^{0}+\sigma_{i j}$ can be found from Eqs. (3.1) or (3.2). Now we can consider a homogeneous body with a continuous displacement field. The average strain and average stress fields can be written as $\bar{\varepsilon}_{i j}=\frac{1}{\Omega} \int_{\Omega} \varepsilon_{i j} d \Omega$ and $\bar{\sigma}_{i j}=\frac{1}{\Omega} \int_{\Omega} \sigma_{i j} d \Omega$, respectively. If we have a twophase medium the average strain field can be written as

$$
\bar{\varepsilon}_{i j}=c_{1} \bar{\varepsilon}_{i j}^{(1)}+c_{2} \bar{\varepsilon}_{i j}^{(2)}
$$

where $\bar{\varepsilon}_{i j}^{(1)}=\frac{1}{\Omega-\Omega_{r}} \int_{\Omega-Q_{r}} \varepsilon_{i j} d \Omega, \quad \bar{\varepsilon}_{i j}^{(2)}=\frac{1}{\Omega_{r}} \int_{Q_{r}} \varepsilon_{i j} d \Omega, c_{1}=\frac{\Omega-\Omega_{r}}{\Omega}$, and $c_{2}=\frac{\Omega_{r}}{\Omega}$. Similarly, the average stress field can be written as

$$
\bar{\sigma}_{i j}=c_{1} \bar{\sigma}_{i j}^{(1)}+c_{2} \bar{\sigma}_{i j}^{(2)}
$$

where $\bar{\sigma}_{i j}^{(1)}=\frac{1}{\Omega-\Omega_{r}} \int_{Q-\Omega,} \sigma_{i j} d Q, \bar{\sigma}_{i j}^{(2)}=\frac{1}{\Omega_{r}} \int_{Q_{r}} \sigma_{i j} d \Omega$.

We may combine Eqs. (3.7) and (3.8) with the prescribed boundary conditions

$$
\bar{\varepsilon}_{i j}=\varepsilon_{i j}^{0}
$$

to obtain

$$
\begin{aligned}
\bar{\sigma}_{i j} & =c_{1}\left(C_{i j k l} \bar{\varepsilon}_{k l}^{(1)}\right)+c_{2}\left(C_{i j k l}^{*} \bar{\varepsilon}_{k l}^{(2)}\right) \\
& =C_{i j k l}\left(\bar{\varepsilon}_{k l}-c_{2} \bar{\varepsilon}_{k l}^{(2)}\right)+c_{2}\left(C_{i j k l}^{*} \bar{\varepsilon}_{k l}^{(2)}\right) \\
& =C_{i j k l} \varepsilon_{k l}^{0}+c_{2}\left(C_{i j k l}^{*}-C_{i j k l}\right) \bar{\varepsilon}_{k l}^{(2)}
\end{aligned}
$$

The effective stiffness $\bar{C}_{i j k l}$ is defined as $\bar{\sigma}_{i j}=\bar{C}_{i j k l} \bar{\varepsilon}_{k l}$, and therefore, if $\bar{\varepsilon}_{i j}^{(2)}$ is related to $\bar{\varepsilon}_{i j}$ by $\bar{\varepsilon}_{i j}^{(2)}=\bar{A}_{i j k l} \varepsilon_{k l}^{0}$, then $\bar{C}_{i j k l}=C_{i j k l}+c_{2}\left(C_{i j m n}^{*}-C_{i j m n}\right) \bar{A}_{m n k l}$. For the isotropic 
materials, $\bar{C}_{l j k l}$ can be written as

$$
\begin{aligned}
& \bar{\mu}=\mu+c_{2}\left(\mu^{*}-\mu\right) \bar{\alpha} \\
& \bar{K}=K+c_{2}\left(K^{*}-K\right) \bar{\beta}
\end{aligned}
$$

\subsection{Solution for Material with Low Concentration of Inclusions}

For small concentrations, we can determine $\bar{\alpha}$ and $\bar{\beta}$ from the work of Eshelby [16]. Recall that $\varepsilon_{k l}^{(2)}=\varepsilon_{k l}^{0}+\varepsilon_{k l}$ is the total strain in $Q_{r}$, and the strain is uniform in the spherical inclusion, $\bar{\varepsilon}_{k l}^{(2)}=\varepsilon_{k l}^{(2)}$. According to Eshelby [16], the eigenstrain and disturbance strain in spherical inclusion can be written as follows:

For $i \neq j$,

$$
\begin{gathered}
\varepsilon_{i j}^{*}=\frac{15\left(\mu^{*}-\mu\right)(1-v)}{(5 v-7) \mu-(8-10 v) \mu^{*}} \varepsilon_{i j}^{0} \\
\varepsilon_{i j}=\frac{(8-10 v)}{15(1-v)} \varepsilon_{i j}^{*}=\frac{(8-10 v)\left(\mu^{*}-\mu\right)}{(5 v-7) \mu-(8-10 v) \mu^{*}} \varepsilon_{i j}^{0}=(\bar{\alpha}-1) \varepsilon_{i j}^{0}
\end{gathered}
$$

For the volume strains,

$$
\begin{aligned}
& \varepsilon_{k k}^{*}=\frac{3(1-v)\left(K^{*}-K\right)}{(4 v-2) K-(1+v) K^{*}} \varepsilon_{k k}^{0}, \\
& \varepsilon_{k k}=\frac{(1+v)}{3(1-v)} \varepsilon_{k k}^{*}=\frac{(1+v)\left(K^{*}-K\right)}{(4 v-2) K-(1+v) K^{*}} \varepsilon_{k k}^{0}=(\bar{\beta}-1) \varepsilon_{k k}^{0}
\end{aligned}
$$

Replacing $\nu$ by $\frac{3 K-2 \mu}{6 K+2 \mu}$, we may substitute $\bar{\alpha}$ and $\bar{\beta}$ obtained from the above expressions into Eq. (3.11), and find

$$
\begin{aligned}
& \bar{\mu}=\mu+\frac{c_{2}\left(\mu^{*}-\mu\right)(15 K+20 \mu) \mu}{(9 K+8 \mu) \mu+(6 K+12 \mu) \mu^{*}} \\
& \bar{K}=K+\frac{c_{2}\left(K^{*}-K\right)(4 \mu+3 K)}{4 \mu+3 K^{*}}
\end{aligned}
$$

\subsection{Solution for Materials with High Concentration of Inclusion or High Porosity}

In the case of a high concentration of inclusions, the concept of an infinite matrix in the composite domain cannot be used. We must, instead, assume a finite subdomain containing the inclusion lumped and originating at a central position, maintaining the same volume ratio as in the original composite material. These subdomains are evenly distributed throughout the whole domain. In the classical analysis, if the matrix is infinite, then the disturbance stress and strain due to the inhomogeneity will tend asymptotically to zero at the outer boundary, but in the finite domain they must satisfy the boundary condition of the sub-domain, namely, $u_{i}=0$ on boundary $S$ for the prescribed iso-strain condition. In order to satisfy the boundary condition of the 
prescribed iso-strain condition, the inhomogeneity inclusion induced disturbancedisplacement field, $u_{i}$, on the boundary $S$ should be eliminated; this can be done by adding the same disturbance-displacement field but opposite sign onto the boundary $S$.

From Eshelby equivalent inclusion method [16] and Love [17], we can calculate the displacement field in the matrix caused by the eigenstrain, $\varepsilon_{i j}^{*}$, and it can be written as

$$
u_{i}=\frac{1}{8 \pi(1-v)} \varepsilon_{j k}^{*} \Phi_{i j k}-\frac{1}{2 \pi} \varepsilon_{i k}^{*} \Phi_{, k}-\frac{v}{4 \pi(1-v)} \varepsilon_{k k}^{*} \Phi_{, i}
$$

where $\Phi$ and $\Psi$ are defined as

$$
\Phi(r)=\int_{Q_{r}} \frac{d \Omega}{\left|r-r^{\prime}\right|}, \Psi(r)=\int_{\Omega,}\left|r-r^{\prime}\right| d \Omega
$$

In order to determine the disturbance-displacement field on the outer boundary $\mathcal{S}$, we evaluate the displacement field given by Eq. (3.15) at large $r$. The result is

$$
u_{i}(r) \approx \frac{\Omega_{r} \varepsilon_{k l}^{*} \varepsilon_{i k l}}{8 \pi(1-v) r^{2}}
$$

where $Q_{r}=\frac{4}{3} \pi r_{1}^{3}, g_{i k l}=(1-2 v)\left(\delta_{i k} l_{l}+\delta_{i l} l_{k}-\delta_{k l} l_{i}\right)+3 l_{i} l_{k} l_{l}, l_{i}=x_{i} / r . r_{1}$ is the radius of inclusion. Substituting for $\Omega_{r}, g_{i k l}$, and $l_{i}$ into Eq. (3.17), $u_{i}$ can be written as

$$
u_{i} \approx \frac{r_{1}^{3} \varepsilon_{k l}^{*}}{6(1-v)}\left[(1-2 v)\left(\delta_{i k} \frac{x_{l}}{r^{3}}+\delta_{i l} \frac{x_{k}}{r^{3}}-\delta_{k l} \frac{x_{i}}{r^{3}}\right)+3 \frac{x_{i} x_{k} x_{l}}{r^{5}}\right]
$$

The inhomogeneity inclusion induced disturbance-strain field, $\varepsilon_{i j}$, on the surface $S$, can be written as follows: $\varepsilon_{i j}=\frac{1}{2}\left(u_{i, j}+u_{j, i}\right)$, where $u_{i, j}$ can be obtained by differentiating Eq. (3.18) with respect to $x_{j}$, and can be written as

$$
\begin{aligned}
u_{i, j} \approx & \frac{r_{1}^{3} \varepsilon_{k l}^{*}}{6(1-v)}\left\{(1-2 v)\left[\left(\frac{\delta_{i k} \delta_{j l}}{r^{3}}+\frac{\delta_{i l} \delta_{j k}}{r^{3}}-\frac{\delta_{i j} \delta_{k l}}{r^{3}}\right)-3\left(\frac{\delta_{i k} x_{j} x_{l}}{r^{5}}+\frac{\delta_{i l} x_{j} x_{k}}{r^{5}}-\frac{\delta_{k l} x_{i} x_{j}}{r^{5}}\right)\right]\right. \\
& \left.+\frac{3}{r^{5}}\left(\delta_{i j} x_{k} x_{l}+\delta_{j k} x_{i} x_{l}+\delta_{j l} x_{i} x_{k}\right)-\frac{15}{r^{7}} x_{i} x_{j} x_{k} x_{l}\right\}
\end{aligned}
$$

Substituting $u_{i, j}$ and $u_{j, i}$ into $\varepsilon_{i j}$, we can get

$$
\begin{aligned}
\varepsilon_{i j} \approx & \frac{r_{1}^{3}}{6(1-v) r^{3}}\left\{(1-2 v)\left\{2 \varepsilon_{i j}^{*}-\delta_{i j} \varepsilon_{k k}^{*}-3\left(l_{i} l_{k} \varepsilon_{j k}^{*}+l_{j} l_{k} \varepsilon_{i k}^{*}-l_{i} l_{j} \varepsilon_{k k}^{*}\right)\right]\right. \\
& \left.+3\left(\delta_{i j} l_{k} l_{l} \varepsilon_{k l}^{*}+l_{i} l_{k} \varepsilon_{j k}^{*}+l_{j} l_{k} \varepsilon_{i k}^{*}\right)-15 l_{i} l_{j} l_{k} l_{l} \varepsilon_{k l}^{*}\right\}
\end{aligned}
$$

In order to satisfy the boundary condition $u_{i}(\Omega)=0$, the displacement field $-u_{i}$ will be added onto the boundary $S$, and the corresponding strain field $-\tilde{\varepsilon}_{i j}$ will be generated 
on the surface $S$. This artificial correction field on the boundary $S$ will then alter the final strain field within the inclusion.

The average strain field in the matrix field induced by this correction field is zero, demonstrated by integrating Eq. (3.20) over the spherical boundary within the matrix, and the resulting average is zero. Thus this correction field within the matrix field will not contribute to the final effective material property change.

In order to obtain a quantitative estimation of shear disturbance-strain $\varepsilon_{i j}(i \neq j)$ on the outer boundary $S$, induced by eigenstrain $\varepsilon_{i j}^{*}$ in the inclusion, we chose $i=1, j=2$,. We are focused here on what the shear disturbance $\varepsilon_{12}$ is on the outer boundary $S$ in term of eigenstrain $\varepsilon_{12}^{*}$. On the boundary $S, r=r_{2}$. Substituting the above into Eq. (3.20), we can get

$$
\varepsilon_{12}(S) \approx \frac{r_{1}^{3}}{6(1-v) r_{2}^{3}}\left\{2(1-2 v)+6 v\left(l_{1}^{2}+l_{2}^{2}\right)-30 l_{1}^{2} l_{2}^{2}\right\} \varepsilon_{12}^{*} \approx \tilde{R}_{1} \varepsilon_{12}^{*}=\tilde{\varepsilon}_{12}(S)
$$

Here, $\tilde{R}_{1}$ can be considered as a transmitting factor, which represents the disturbance on the outer surface $S$ caused by the unit eigenstrain, $\varepsilon_{12}^{*}$, in the inclusion.

One assumption was made here: the transmitting factor induced by the correction field, from the boundary surface $S$ to the inclusion, is the same as $\tilde{R}$. This is based on the first-order approximation with a relatively large volume fraction of inclusion. The similar analogy of the wave propagation through the media can be used to demonstrate the validity of the above assumption. For example, a shear wave travels from the source media to the fixed-rigid boundary, which has much higher shear modulus and bulk modulus compared to that of wave source media. After the shear wave reaches the fixed boundary, the reflected shear wave will have the same initial speed as that of the incoming shear wave before reaching the fixed boundary. Here, the fixed boundary is equivalent to the boundary condition with $u_{i}(\Omega)=0$. The transmitting factor, either from the fixed boundary to source or from source to the fixed boundary, will remain constant as long as the material properties of the source media remain constant.

Thus the induced strain field, $\tilde{\varepsilon}_{i j}^{f}$ on the interface of inclusion and matrix, $S_{12}$, by the correction field applied on the boundary $S$ can be written as

$$
\tilde{\varepsilon}_{12}^{f}=-\tilde{R}_{1} \tilde{\varepsilon}_{12}(S)=-\tilde{R}_{1}^{2} \varepsilon_{12}^{*}=-\left|\tilde{R}_{1}\right|^{2} \varepsilon_{12}^{*}
$$

In order to have a more general transmitting factor, we define a mean magnitude of the transmitting factor, $\tilde{R}$, on the boundary $S$, and is written as follows: 


$$
\tilde{R}=\frac{1}{4 \pi r_{2}^{2}} \int_{S}\left|\tilde{R}_{1}\right| d S
$$

in spherical coordinate, $l_{1}=\sin \theta \cos \phi, l_{2}=\sin \theta \sin \phi, d S=r_{2}^{2} \sin \theta d \theta d \phi$. Substituting the above into Eqs. (3.30) and (3.31), we can get

$$
\tilde{R}=\frac{c_{2}}{24 \pi(1-v)} \int_{0}^{2 \pi} \int_{0}^{\pi}\left|2(1-2 v)+6 v \sin ^{2} \theta-30 \sin ^{4} \theta \cos ^{2} \phi \sin ^{2} \phi\right| \sin \theta d \theta d \phi
$$

where $c_{2}=\left(r_{1} / r_{2}\right)^{3}$ is the volume fraction of the inclusion. By the numerical evaluation and the linear-least-squares fit for $v$ (range from 0 to 0.5 ), the integral part of Eq. (3.24) can be simplified to $(-10.72 v+22.45)$. Substituting the above into Eq. (3.24), the final form of $\tilde{R}$ can be written as

$$
\tilde{R}=\frac{(22.45-10.72 v) c_{2}}{24 \pi(1-v)}=\frac{(0.3-0.14 v) c_{2}}{(1-v)}
$$

Repeating the above procedures, the mean amplitude of transmitting factors can be obtained for the disturbance field, $\varepsilon_{13}$ and $\varepsilon_{23}$, which are identical to the $\tilde{R}$. Thus the estimated strain field, $\bar{\varepsilon}_{i j}^{f}$, on the interface of inclusion and matrix, induced by the correction field applied on the boundary surface, $S$, can be written as

$$
\tilde{\varepsilon}_{i j}^{f}=-\tilde{R}^{2} \varepsilon_{i j}^{*}
$$

Substituting $\tilde{R}, \tilde{\varepsilon}_{i j}$ and $\varepsilon_{i j}^{*}$ into Eq. (3.26), we can get

$$
\tilde{\varepsilon}_{i j}^{f}=-\tilde{R}^{2} \varepsilon_{i j}^{*}=-\left\{\left[\frac{(0.3-0.14 v) c_{2}}{(1-v)}\right]^{2}\left[\frac{15\left(\mu^{*}-\mu\right)(1-v)}{(5 v-7) \mu-(8-10 v) \mu^{*}}\right]\right\} \varepsilon_{i j}^{0}=-R \varepsilon_{i j}^{0}
$$

where $R$ is defined as the modification factor. Substituting $v=\frac{(3 K-2 \mu)}{(6 K+2 \mu)}$ and $\bar{\alpha}$ into Eq. (3.27), $R$ can be written as

$$
R=\left(\frac{(1.38 K+0.88 \mu) c_{2}}{3 K+4 \mu}\right)^{2}\left(\frac{\bar{\alpha}}{\mu}\right)\left(\mu^{*}-\mu\right)
$$

This induced strain field acting on the boundary of inclusion can be written as $-R \varepsilon_{i j}^{0}$. From the linear relationship between the inclusion disturbance $\varepsilon_{i j}$ and $\varepsilon_{i j}^{0}$ as shown in Eq. (3.12), the corresponding disturbance in the inclusion, induced by $-R \varepsilon_{i j}^{0}$, can be written as $-R \varepsilon_{i j}$. The net disturbance in the inclusion, after the corrections, is $(1-R) \varepsilon_{i j},-1<R<1$. Thus the final modification of $\bar{\varepsilon}_{i j}^{(2)}$ can be written as

$$
\bar{\varepsilon}_{i j}^{(2)}=\varepsilon_{i j}^{0}+(1-R) \varepsilon_{i j}
$$

Following the same analogy as for the low-concentration case, the effective stiffness, $\mu$, for isotropic materials can be written as 


$$
\bar{\mu}=\mu+c_{2}\left(\mu^{*}-\mu\right) \bar{\alpha}^{\mu}
$$

where $\overline{\boldsymbol{a}}^{M}$ can be written as

$$
\bar{\alpha}^{M}=1+(1-R)(\bar{\alpha}-1)=\bar{\alpha}(1-R)+R
$$

For the case of a very low concentration, $c_{2}$ will tend to zero, and the modification factor $R \approx 0$. This will result in $\bar{\alpha}^{M}=\bar{\alpha}$; thus Eq. (3.30) will be equivalent to Eq. (3.11) for the low-concentration case.

From Eq. (3.15), we find $u_{i}$ for pure dilatation to be

$$
u_{i}=-\frac{(1+v)}{12 \pi(1-v)} e_{k k}^{*} \Phi_{, i}
$$

and the volume strain can be written as

$$
\varepsilon_{k k}=u_{i, i}=-\frac{1+v)}{12 \pi(1-v)} \Phi_{, i i} \varepsilon_{k k}^{*}
$$

For large distances from the inclusion, $\Phi \approx \frac{\Omega_{r}}{r}$. We then set $\bar{\Phi}=\frac{Q_{r}}{r}$ and obtain

$$
\bar{\Phi}_{, i}=-\Omega_{r} \frac{x_{i}}{r^{3}}, \quad \bar{\Phi}_{. i i}=\Omega_{r}\left[3\left(\frac{x_{i}}{r^{4}} \cdot \frac{x_{i}}{r}\right)-\frac{3}{r^{3}}\right]=0
$$

Substituting $\bar{\Phi}_{, k k}=0$ into Eq. (3.33) we obtain $\tilde{\varepsilon}_{k k}=0$. This implies that the dilatation in the matrix due to the pure dilatation eigenstrain, $\varepsilon_{k k}^{*}$, is zero (i.e., the disturbance volume strain $\tilde{\varepsilon}_{k k}$ on the boundary $S$ is also zero.) In order to satisfy the boundary condition $u_{i}(S)=0$, the field $-u_{i}$ will be added at the boundary $S$, and this will generate the volume strain field $-\tilde{\varepsilon}_{k k}$ on the boundary $S$, which is equal to zero. Thus the induced volume strain, $\varepsilon_{k k}$, of inclusion due to the added field will also equal zero. There is no modification for the final volume strain field $\varepsilon_{k k}$ in the inclusion. Therefore, the effective bulk modulus, $\tilde{K}$, remains the same as Eq. (3.14). Hereafter, the derived effective stiffnesses

$$
\begin{aligned}
& \bar{\mu}=\mu+c_{2}\left(\mu^{*}-\mu\right) \bar{\alpha}^{N} \\
& \bar{K}=K+c_{2}\left(K^{*}-K\right) \bar{\beta}
\end{aligned}
$$

will be used for both low and high concentration throughout the rest of the paper.

\subsection{Comparison of the Modified Direct Method Prediction with Other Methods}

3.5.1 Effect of Young's Modulus of Glass Microspheres in Polyester Matrix (For Hard Inclusion Case)

Experimental data for a composite system with a dispersion of solid microspheres 
embedded in a polyester matrix have been given by Richard [18]. The glass microspheres were soda lime glass spheres ranging from 210 to 297 microns in diameter. A series of tensile tests were performed to characterize the response of the composite with changes in the volume fraction of glass. The measurements are of the effective uniaxial modulus $E$. For purposes of comparing the data with the predictions of modified direct method, the effective shear modulus from Eq. (3.30) and the effective bulk modulus from Eq. (3.14) have been combined to give $E=\frac{9 K_{\mu}}{3 K+\mu}$. The exact solutions derived by Christensen and Lo [19] were also included in the data comparisons, where the effective shear modulus of exact solution was from Eq. (4.12) of reference 19, and the effective bulk modulus was from Eq. (3.17) of reference 19. The results are shown in Figure 1, and the following material properties were used in the analysis:

$\mu_{0}=0.8473 \cdot 10^{2} \mathrm{ksi}, \quad K_{0}=7.280 \cdot 10^{2} \mathrm{ksi}, \quad v_{0}=0.444, \quad E_{0}=2.447 \cdot 10^{2} \mathrm{ksi}$

: $\mu_{i}=42.148 \cdot 10^{2} \mathrm{ksi}, \quad K_{i}=58.62 \cdot 10^{2} \mathrm{ksi}, \quad v_{i}=0.210, E_{i}=102.0 \cdot 10^{2} \mathrm{ksi}$ Figure 1 shows that both the modified direct method and the exact solution from Christensen and Lo have good agreement with experimental data for relatively low concentration; however, the modified direct method gives better predictions for higher concentration.

\subsubsection{Effect of Porosity on Compressibility of Glass (For Soft Inclusion Case)}

The effect of voids or fluid inclusions on the elastic moduli can be estimated by letting the moduli of the inclusion vanish in the expression for the two-phase composites. This will cause a stress-concentration effect near the surface of the inclusion, especially for the high-porosity case. Here we adopt an iteration scheme to decrease the interaction effect and not overestimate the effect of pore-type inclusions at higher porosities. The iteration scheme is as follows: when we calculate the effective material properties at one specific low porosity, we then update the effective bulk modulus $K$ and the effective shear modulus $\mu$ at this state, and then go to the next higher porosity state. Therefore, we update $K, \mu$ at each state in the series. This scheme is equivalent to the differential scheme.

We compare the prediction of the modified direct method with the experimental result found by Walsh and Brace [20] for porous glass, where $K_{0} / \mu_{0}=1.518, v_{0}=$ 0.23. From Figure 2 we can see the data are located near the self-consistent curve for porosity less than 0.20. For higher porosity, the Kuster-Toksöz method [21] and Christensen's exact solution fit the data slightly better than the new self-consistent method [22] but are still not ideal. However, the modified direct method fits the data very well.

\section{Experimental Studies}

In order to verify the theory developed in Section 3 and its application to aggregate 


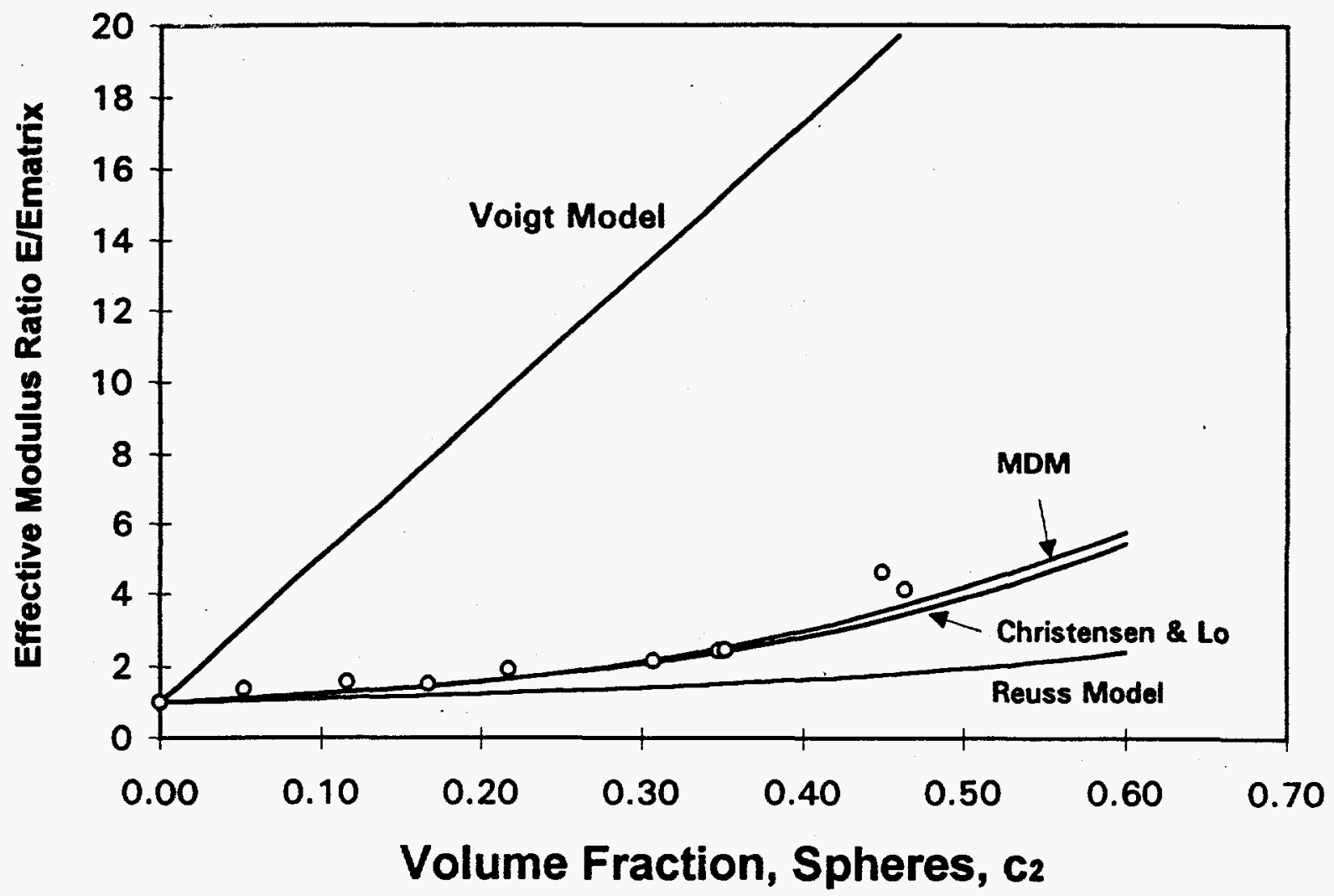

Figure 1. Effective Young's Modulus, Glass Spheres in Polyster Matrix, the Modified Direct Method (MDM).

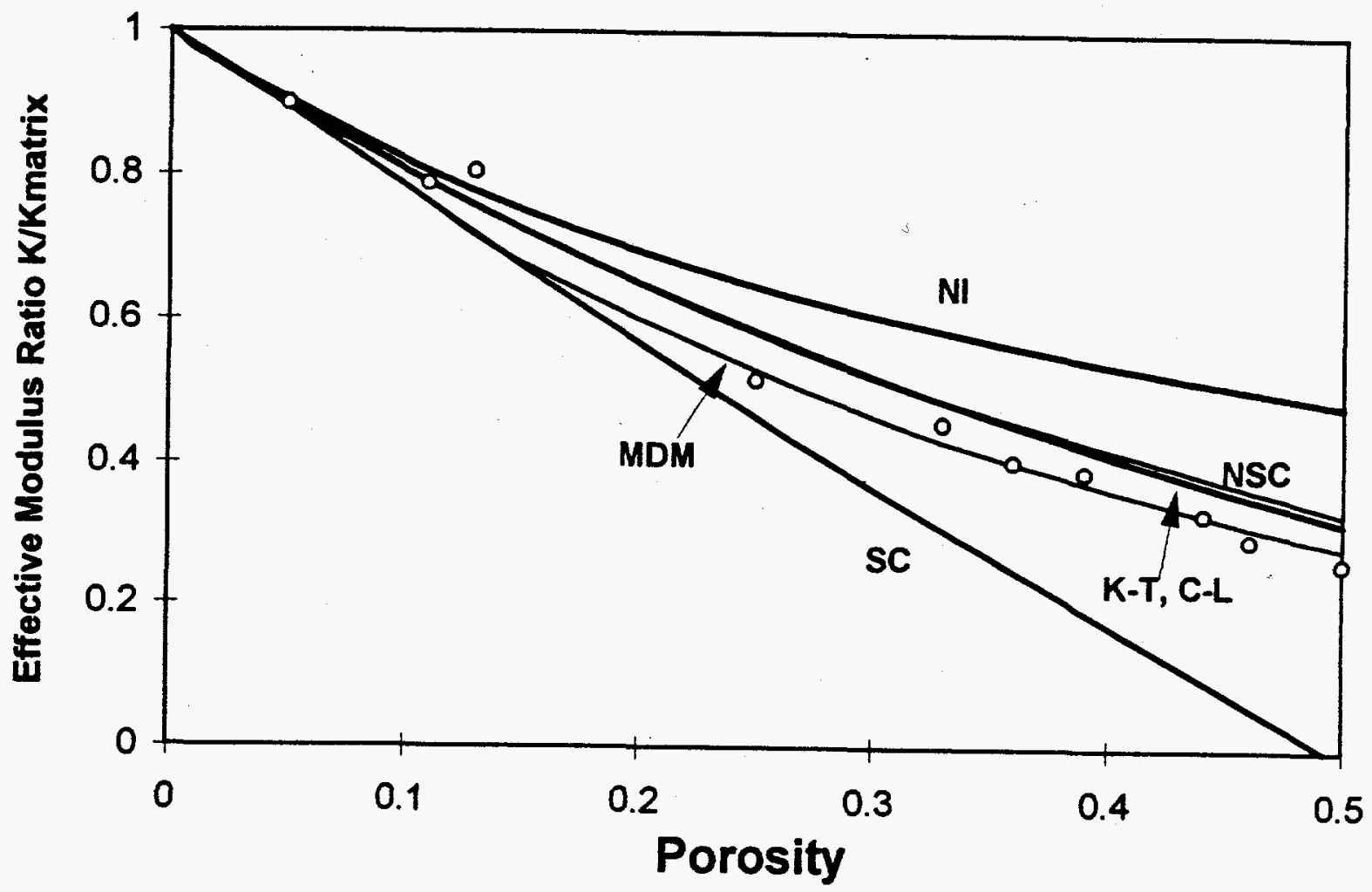

Figure 2. Bulk modulus of porous glass, self-consistent (SC), modified direct method(MDM), Kuster-Toksoz (K-T), Christensen-Lo (C-L), new self-consistent (NSC), and no-interaction (NI). 
composites, some experiments were carried out. The velocities of elastic waves in cement paste and mortar under various conditions were determined experimentally. The elastic properties were determined by measuring the acoustic velocities, $V_{P}$ and $V_{s}$, in a 2-in.-cubic specimen. Once the wave speeds and density are known, then the elastic moduli can be determined directly from the following relations:

$$
K=\rho\left(V_{P}^{2}-\frac{4}{3} V_{s}^{2}\right), \mu=\rho V_{S}^{2}
$$

The test procedure and test specimens are discussed in detail elsewhere [23]. For pure cement paste specimens, the following water-cement ratios were used: $0.20,0.25,0.30$, $0.35,0.40,0.45$. For mortar specimens, the water-cement ratio was kept constant at 0.30 , and sand concentrations were used at $0 \%, 15 \%, 27 \%, 40 \%, 52 \%$, and $65 \%$.

\subsection{Saturated Cement Paste}

Since the elastic moduli of $0 \%$ porosity cement paste are unknown, the properties of the matrix are used from one of the specimens as the starting matrix. Here, the properties of matrix are defined by means of the results of tests on the specimens with $20 \%$ water-cement ratio. This gives $\mu_{m}=13.87 \mathrm{GPa}, K_{m}=25.95 \mathrm{GPa}$, and $\rho=2278$ $\mathrm{kg} / \mathrm{m}^{3}$. Since the specimens were saturated with water, the inclusion phase will be water. This has the properties $K_{W}=2.0 \mathrm{GPa}, \mu_{W}=0.0 \mathrm{GPa}$, and $\rho_{W}=1000 \mathrm{~kg} / \mathrm{m}^{3}$. Figure 3 shows that the modified direct method has much better fits of the experimental data than that of Christensen's method. The elastic moduli of the original cement paste are unknown because it is not possible to prepare a specimen with $0 \%$ porosity. We use the modified direct method with an optimization procedure to predict the elastic moduli of cement paste at $0 \%$ porosity, and the determined moduli are listed below

$$
\mu=24.67 \mathrm{GPa}, K=51.45 \mathrm{GPa}
$$

\subsection{Saturated Mortar}

In this case, one takes the porous, water-saturated mortar to be the matrix phase, and takes the sand grains as the inclusions. The properties of the matrix are defined by means of the results of tests on the specimens with zero sand content. This gives $\mu_{m}=11.8 \mathrm{GPa}, K_{m}=22.4 \mathrm{GPa}$, and $\rho=2182 \mathrm{~kg} / \mathrm{m}^{3}$. The properties of the silica grains are $\mu_{s}=37 \mathrm{GPa}, K_{s}=44 \mathrm{GPa}$, and $\rho_{s}=2700 \mathrm{~kg} / \mathrm{m}^{3}$. The five pairs of wave speeds are shown in Figure 4, along with predictions corresponding to the Reuss and Voigt bounds and Christensen's models. The modified direct method has the best fits of the experimental data for the entire range of sand concentrations from $0 \%$ to $65 \%$.

\section{Conclusions}

From the research that has been done for composite materials, it is evident that in spite of the great amount of work that has been done on composite modeling, there is 


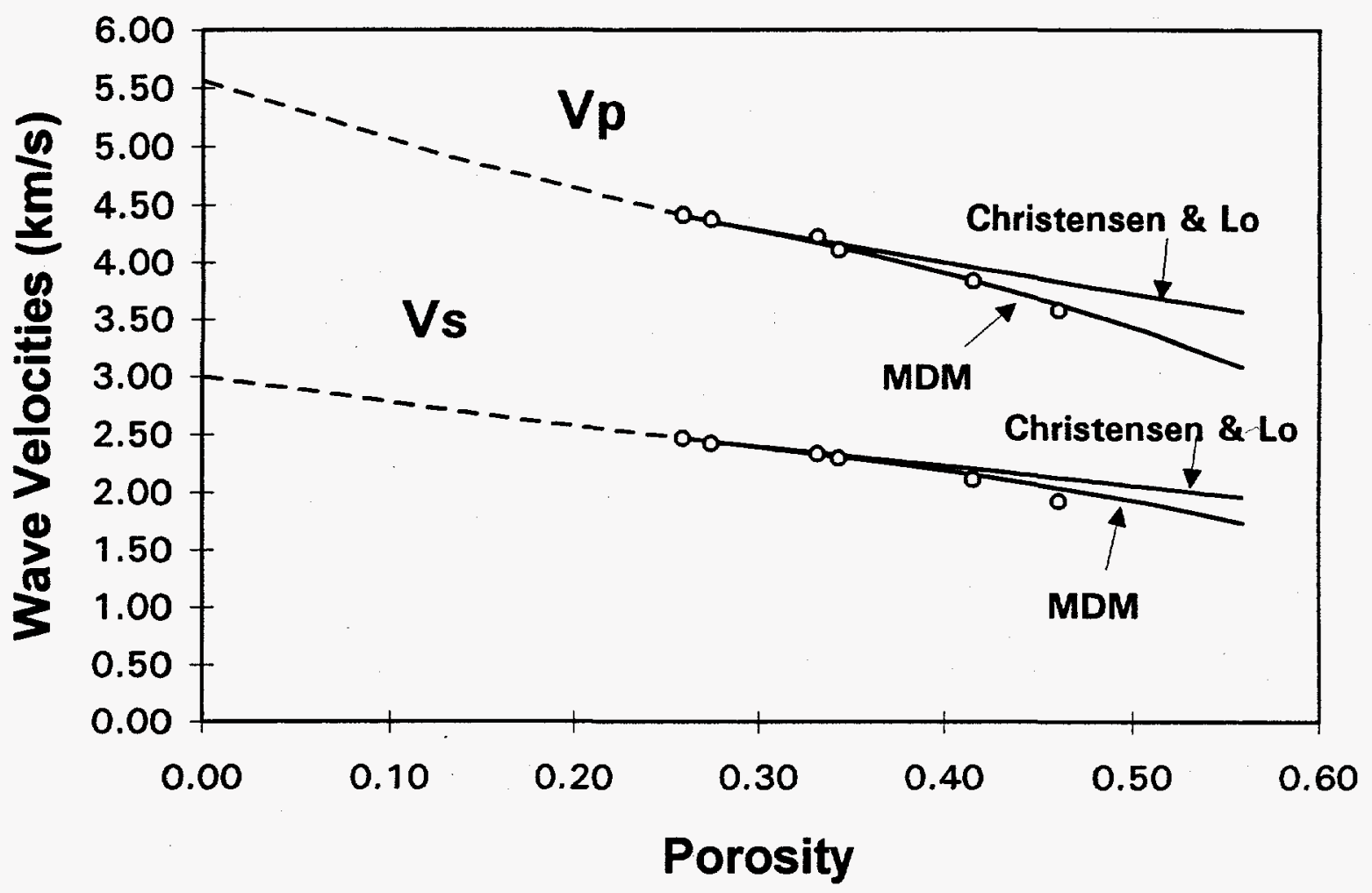

Figure 3. Wave Velocities in Saturated Cement Paste as Function of Porosity

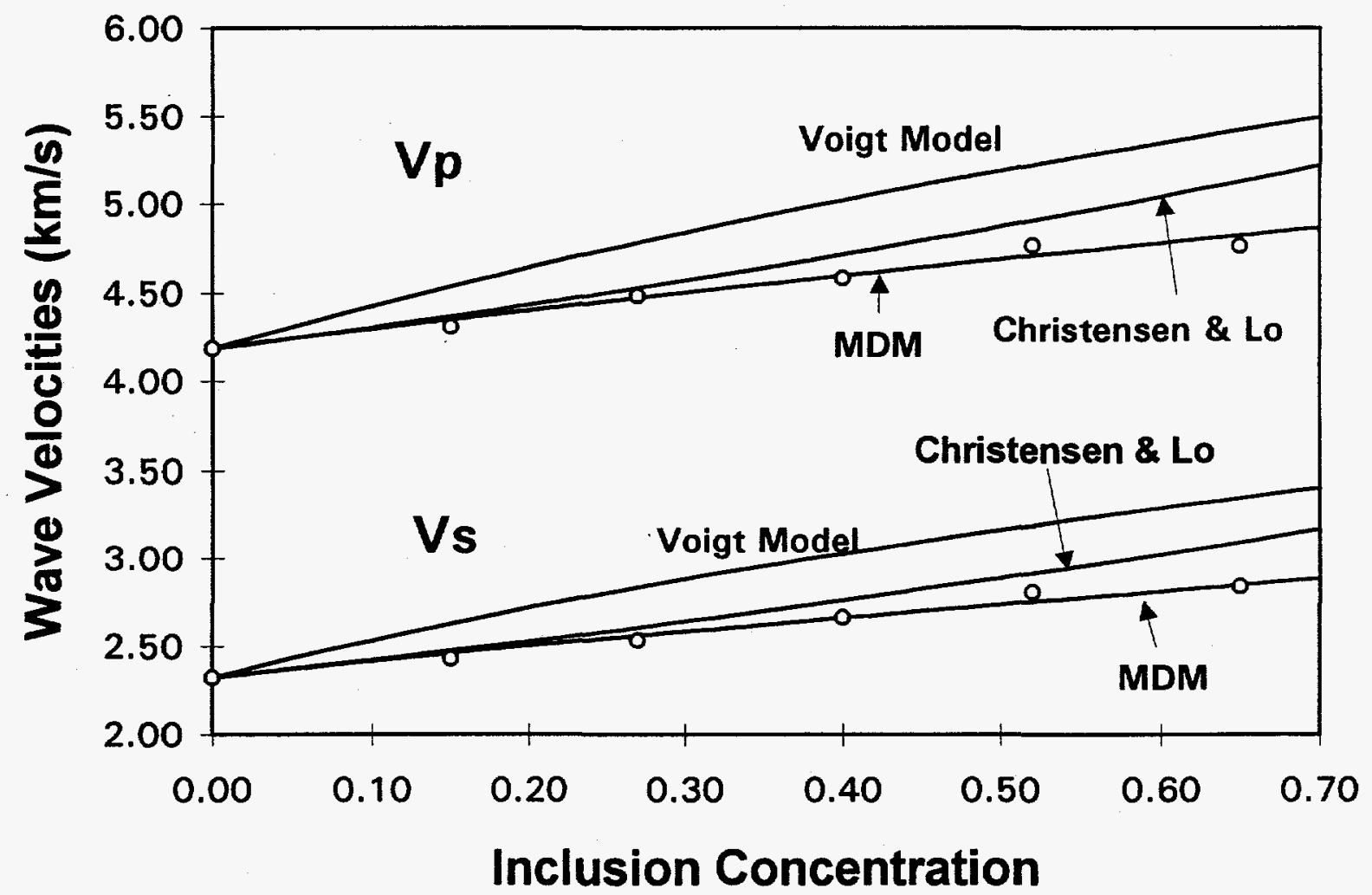

Figure 4. Wave Velocities in Saturated Mortar as a Function of Sand Concentration. 
much that remains to be done, such as interfacial phenomena, debonding and contacting inclusions, phase geometry, etc. Due to the complexity of composite materials, existing theories are often unrealistic because exact analytical theory needs detailed modeling of the various factors. The modeling of these factors is not always available and then the effective bounds are usually too wide for practical application. For engineering purposes a more practical and general model is desired. The modified direct method was developed to approach the above requirements. The modified direct method is a scheme for the estimation of elastic moduli of composite materials and is based on micromechanical theory and classical elasticity. Using the statistical homogeneous assumption and the two-phase composite approach, one takes the average field of the composite. In this analysis, we use the spherical shape inclusion, and we assume that the phases are ideally elastic and homogeneous over the whole of their bulk. Also the adhesion between phases is perfect; and any other form of interaction is absent. Comparison with experimental data and with other well-known methods, such as Christensen-Lo, Kuster-Toksöz, and the self-consistent method, shows that the modified direct method provides a very good estimation of the elastic moduli in different kinds of problems, such as the soft and hard inclusion cases, porous materials, at various concentrations and/or various porosities.

\section{References}

1. Bland, D.R. (1960). The Theory of Linear Viscoelasticity. Pergamon Press, Oxford, London.

2. Tobolsky, A.V. (1956). "Stress Relaxation of the Viscoelastic Properties of Polymers." Journal of Applied Physics, Vol. 27, pp. 673-685.

3. Timoshenko, S., and Goodier, J.N. (1969). Theory of Elasticity, Mcgraw-Hill.

4. Hashin, Z. (1970). "Mechanics of Composite Materials." Proceedings, 5th Symposium, Naval Structure Mechanics.

5. Wu, I.N., and Sendeckyj, G.P. (1974). "Multiple Circular Inclusion Problems in Plane Elastostatics." Journal of Applied Mechanics, Vol. 41, ser. E, pp. 215-221.

6. Goree, J.G., and Wilson, H.B. (1967). "Axisymmetric Torsional Stresses in a Solid Containing Two Partially Bonded Rigid Spherical Inclusions. " Journal of Applied Mechanics, Vol. 34, ser. E, pp. 313-320.

7. Theocaris, P.S., and Paipetis, S.A. (1978). "Indentation Studies in AluminumFilled Epoxies." Journal of Applied Ploymer Science, Vol. 22, pp. 2245-2252.

8. Papanicolaou, G.C., and Paipetis, S.A. (1978). "The Concept of Boundary Interphase in Composite." Colloid and Polymer Science, Vol. 256, pp. 625-630. 
9. Wilson, H.B., and Hashin, Z. (1962). "The Elastic Moduli of Heterogeneous Materials." Journal of Applied Mechanics, Vol. 29, pp. 143-150.

10. Budiansky, B. (1965). "On the Elastic Moduli of Some Heterogeneous Materials." Journal of Mechanics Physics Solids, Vol. 13, pp. 223-227.

11. Hill, R.A. (1965). "Self Consistent Mechanics of Composite Materials." Journal of Mechanics Physics Solids, Vol. 13, pp. 213-222.

12. Walpole, L.J. (1969). "On the Overall Elastic Moduli of Composite Materials." Journal of Mechanics Physics Solids, Vol. 17, pp. 235-251.

13. Hashin, Z. (1964). "Theory of Mechanical Behavior of Heterogeneous Media." Applied Mechanics Reviews, Vol. 17, pp. 1-9.

14. Paul, B. (1960). "Prediction of the Elastic Constants of Multiphase Materials." Transactions AIME, Vol. 218, pp. 36-41.

15. Hill, R. (1963). "New Derivation of Some Elastic Extremum Principles." Progress in Applied Mechanics, the Prager Anniversary Volume, MacMillan, New York, pp. 99-106.

16. Eshelby, J.D. (1957). "The Determination of the Elastic Field of an Ellipsoidal Inclusion." Royal Society London Ser. A., Vol. 241, pp. 376-396.

17. Love, A.E.H. (1954). Mathematical Theory of Elasticity, Cambridge University.

18. Richard, T.G. (1975). "The Mechanical Behavior of a Solid Microsphere Filled Composite." Journal of Composite Material, Vol. 9, pp. 108.

19. Christensen, R.M. (1979). Mechanics of Composite Materials, John Wiley \& Sons, New York.

20. Walsh, J.B., and Brace, W.F. (1965). "The Effect of Porosity on Compressibility of Glass." Journal of American Ceramic Society, Vol. 48, pp. 605-608.

21. Kuster, G., and Toksöz, (1974). "Velocity and Attenuation of Seismic Waves in Two Phase Media." Geophysics, Vol. 39, pp. 487-506.

22. Henyey, F.S., and Pomphrey, N. (1982). "Self-Consistent Elastic Moduli of a Cracked Solid." Geophysics Res. Letter, Vol. 9, pp. 903-906.

23. Wang, J.A., Lubliner, J., and Monteiro, P.J. (1989). "The Effect of Ice Formation on the Elastic Moduli of Porous Media." Cement and Concrete Research, Vol. 18, pp. 874-889. 